\title{
General Relativity and Quantum Gravity in Terms of Quantum Measure: A philosophical comment
}

\begin{abstract}
The paper discusses the philosophical conclusions, which the interrelation between quantum mechanics and general relativity implies by quantum measure.

Quantum measure is three-dimensional, both universal as the Borel measure and complete as the Lebesgue one. Its unit is a quantum bit (qubit) and can be considered as a generalization of the unit of classical information, a bit. It allows quantum mechanics to be interpreted in terms of quantum information, and all physical processes to be seen as informational in a generalized sense. This implies a fundamental connection between the physical and material, on the one hand, and the mathematical and ideal, on the other hand. Quantum measure unifies them by a common and joint informational unit.

Furthermore the approach clears up philosophically how quantum mechanics and general relativity can be understood correspondingly as the holistic and temporal aspect of one and the same, the state of a quantum system, e.g. that of the universe as a whole. The key link between them is the notion of the Bekenstein bound as well as that of quantum temperature. General relativity can be interpreted as a special particular case of quantum gravity. All principles underlain by Einstein (1918) reduce the latter to the former. Consequently their generalization and therefore violation addresses directly a theory of quantum gravity.

Quantum measure reinterprets newly the "Bing Bang" theories about the beginning of the universe. It measures jointly any quantum leap and smooth motion complementary to each other and thus, the jump-like initiation of anything and the corresponding continuous process of its appearance. Quantum measure unifies the "Big Bang" and the whole visible expansion of the universe as two complementary "halves" of one and the same, the set of all states of the universe as a whole. It is a scientific viewpoint to the "creation from nothing".
\end{abstract}




\section{General Relativity and Quantum Gravity in Terms of Quantum Measure: A philosophical comment}

\section{Table of contents:}

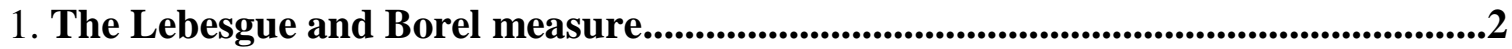

2. The quantum measure and its construction............................................................3

3. The quantum measure compared with the Lebesgue and Borel measure.................7

4. The origin of quantum measure....................................................................................................8

5. Physical quantity measured by quantum measure...............................................14

6. Quantum measure and quantum quantity in terms of the Bekenstein bound.......16

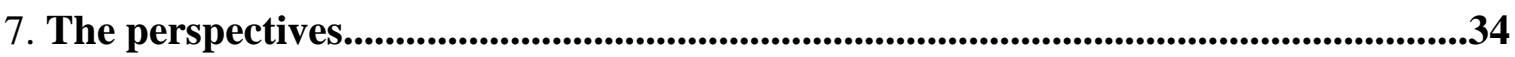

References..............................................................................................................................35

\section{List of Tables}

Table 1: From LM and BM to QM........................................................................15

Table 2: Table 1 paraphrased...............................................................................................15

Table 3: Table 1 and Table 2 unified..................................................................................15

The main question is how nothing (pure probability) can turn into something (physical quantity). The best idea is that both have a common measure. This problem is resolved implicitly in quantum mechanics introducing the formalism of wave functions, which are points in Hilbert space. Its approach can be equivalently represented explicitly in terms of quantum measure, i.e. by the notion of "qubit" defined and utilized in the theory of quantum information. The paper addresses the corresponding philosophical interpretation focused on the interrelation of quantum mechanics and general relativity, and thus on the problem of quantum gravity from a methodological viewpoint.

\section{The Lebesgue (LM) and Borel (BM) measure}

They will be discussed in relation to the axiom of choice ("AC", "NAC" = no axiom of choice) and the continuum hypothesis ("CH", "NCH" = no continuum hypothesis):

$L M$ and BM coincide (AC, $\mathrm{CH}$ ) Carathéodory's extension theorem as to Borel sets (BS) according to Carathéodory's (1956) extension theorem, and either can be distinguished only unconstructively (AC; $\mathrm{CH}$ or $\mathrm{NCH})$ as to non-Borel sets (NBS) or cannot be juxtaposed at all (NAC; $\mathrm{NCH}$ ). Then one can ascribe whatever difference including no difference. That incomparability is a typical situation in quantum mechanics and it represents the proper content of "complementarity". Here the Lebesgue measure is implied to be one-dimensional since the 
real line is such.

Dimensionality, LM, and BM: One must distinguish the dimensionality of the space being measured from the dimensionality of measure, by which the space is measured. The idea of probability as well as that of number in general is to be introduced a universal measure (quantity), by which all (pears, apples, distances, volumes and all the rest) can be measured as separately, item (quality) by item, as together. BM uses n-dimensional spheres, which it compares in radius independently of the number of dimensions. That radius is the Borel measure, and if it is finite, admits the Kolmogorov probability.

One can suppose, though counterintuitively, the case, where the dimensionality of the space being measured is lesser than that of the measure, and that such a case may have a nonempty intersection with $\mathrm{NCH}$. The conjecture would not make much sense while one does not point out a universal measure of the dimensionality greater than one.

\section{Quantum measure (QM) and its construction}

QM is a three-dimensional universal one. A motivation may be for it to be introduced an as complete (like LM) as universal (like BM) measure. It should resolve the problem for completing BM in general (both AC \& NAC):

An alternative, but equivalent approach is to be measured empty intervals (without any points in them), i.e. discrete or quantum leaps, in the same way as complete intervals of continuum. In fact quantum mechanics is what forced the rising of such a measure (\& probability): look at 5.

Moreover, quantum measure is more complete than LM in a sense or even is the most complete measure known to mankind since it can measure not only infinitely small empty, but any finite and even infinite leaps. However it postpones the question to complete them as no need to do it initially, on the one hand, and the general AC \& NAC invariance even requires for the complete and incomplete case to be equated therefore rejecting the need of completion, on the other. That rather strange state of affairs is discussed in details below.

Given $B M$, the construction of $Q M$ is the following:

The objective is to be measured all the NBS as being reduced into some combination of the following three types partially complete:

NBS complete in relative complement;

NBS complete in countable union;

NBS complete in countable intersection.

A partial measure (or a partial probability as a finite measure) corresponds in each of the three cases above.

If a NBS is incomplete in one or more, or even in all of the three relation above, its corresponding measure(s) [probability (-es)] is (are) accepted as zero.

$\mathrm{BM}$ is the particular case where the three measures (probabilities) coincide. If a NBS is incomplete in any relation, it has a zero BM anyway. That backdoor is substantive for reconciling quantum theory based on QM and general relativity grounded on LM or BM in fact.

That kind of construction will be called tricolor hereinafter. The tricolor has exact 
correspondences in set theory and logic.

Let us now consider as an example of the case of tricolor or quantum probability compared with the classical one. One substitutes the unit ball for the interval of $[0,1]$ :

The unit ball can be decomposed in a "spin" way into two orthogonal circles.

The point of the unit ball generalizes that of $[0,1]$.

The point of the unit ball can be represented equivalently both as the two correlating complex numbers (the two projections on the orthogonal circles) and as three independent numbers (those of the tricolor above).

As the interval of $[0,1]$ allows of introducing the unit of classical information, a bit, as the unit ball does the same for quantum information, a qubit:

Since a bit can be thought as the alternative choice between two points: 0 or 1, a qubit might be thought as the choice of a point of the ball, i.e. as a choice among a continuum of alternatives in final analysis.

The $[0,1]$ is the universal measuring unit of all what can be classically measured. It can be illustrated as a "tape measure" for anything which is something, but not nothing. However the unit ball is a more universal measuring unit since it can measure as anything which is something as nothing in a uniform way. In other words, it can measure as the continuous as the discrete without completing the latter with the continuum of a continuous medium of points, i.e. without transforming nothing in something. Consequently, the unit ball is the perfect measure for quantum mechanics since aids it in resolving its main question, namely: How can nothing (pure probability) become something (physical quantity)?

Many philosophers reckon that the same kind of question, why there is something rather than nothing, is the beginning of philosophy, too. Quantum mechanics gives an answer, which is the single one that mankind has managed to reach and which, fortunately or unfortunately, is constructive besides.

Given $L M$, the construction of $Q M$ is the following:

One builds a tricolor measure as the BM for any dimension.

One might consider a "vector" measure, which components are 3D balls. In fact, it is equivalent both to Minkowski and to Hilbert space. That unit-ball vector represents a unit covariant vector, i.e. just a measure.

Any measure of the ball vector would be QM on LM. If the measure is the usual one of the vector length, the measured result would be a 3D ball rather than a $1 \mathrm{D}$ length. The axiom of choice does not use in that QM-on-LM construction.

Using the axiom of choice, a ball is equivalent to any set of balls, which is known as the Banach and Tarski (1924) paradox. So, one need not construct a ball-vector measure as above since it is directly equal to a ball (i.e. QM) according to the axiom of choice.

The last two paragraphs show the original invariance of QM to the axiom of choice unlike LM and BM. As to BM that invariance is an undecidable statement. One might say that $\mathrm{BM}$ even possesses anyway a specific invariance or universality to the axiom of choice: the invariance of incompleteness: $\mathrm{BM}$ is incomplete as with the axiom of choice as without it. As to 
LM, it is complete without AC, but incomplete with AC: Indeed the construction of a Vitali (1905) set, which is immeasurable by LM, requires necessarily AC. At the same time, the way of its construction shows that any Vitali set is a subset of a null set such as that of all the rational numbers within the interval $[0,1]$ since there is a one-to-one constructing mapping between the Vitali set and that set of the rational numbers. Consequently LM under the condition of AC is incomplete since there is a subset of a null set, which is immeasurable: the Vitali set.

The consideration shows that LM occupies an intermediate position between the complete QM and the incomplete BM being partly complete (without AC) and partly incomplete (with AC). Thus LM can also demonstrate AC as the boundary between potential and actually infinity. LM under condition of AC can measure anything which is finite, but nothing which is infinite. QM unlike it can measure both even under AC.

Thus the invariance of QM to the axiom of choice can be added to the motivation of QM since quantum mechanics needs such invariance: Really, quantum measuring requires the axiom of choice, and any quantum state by itself rejects it being due the "no hidden variables" theorems (Neumann 1932: pp. 167-173; Kochen and Specker 1967). Consequently, the epistemological "equation", which equates any state "by itself" and the result of its measuring, needs that invariance in the case of quantum mechanics.

A problem remains to be solved (as if): Is there a BM or LM, with which no QM corresponds after utilizing the aforesaid procedure? The finite or infinite discrete leaps are described by QM unlike LM and BM: Consequently QM can be accepted as more general. However, are there cases, too, which admit BM or LM, but not QM?

Unfortunately that question is not one of abstract, purely mathematical interest since it is an interpretation of the quantum-gravity problem into the measure-theory language. General relativity uses LM, while quantum mechanics QM. If general relativity is true (as seems) and there is a LM (BM) which is not QM (LM-no-QM), then quantum gravity is an undecidable problem. Vice versa: Quantum gravity is resolvable if and only if QM is more general than (since it cannot be equivalent with) LM (BM).

A try for a short answer might be as follows:

The QM-on-LM construction excludes the LM-no-QM conjecture. However it cannot serve for refusing a nonconstructive proof of LM-no-QM existence in general.

Any pure proof of that kind, which requires necessarily the axiom of choice, can be neglected because of the QM invariance to AC/ NAC.

No other proof of pure LM-no-QM existence can be omitted, but whether there are such ones, no one knows. That pure existence is not only a question of abstract and theoretical interest. It suggests that a more general measure than QM can be ever found on the base of LM-no-QM.

One can suppose a new invariance to $\mathrm{CH} / \mathrm{NCN}$ similar to the $\mathrm{QM}$ invariance to $\mathrm{AC}$ / NAC. In fact, it would be equivalent to the existence of a countable model for any mathematical structure of first order: This is a well-known direct corollary of the Löwenheim-Skolem theorem (Löwenheim 1915; Skolem 1919a, 1919b). Thus that alleged as a new invariance would not 
expand out of QM, though. The reason is that $\mathrm{CH}$ implies $\mathrm{AC}$.

However one can continue the implication of $\mathrm{AC}$ from $\mathrm{CH}$ in the following way: $\mathrm{AC}$ implies Skolem's "paradox" (Skolem 1923): The latter implies the impossibility to be compared infinite powers and that $\mathrm{CH} / \mathrm{NCH}$ is undecidable for the sake of that. That is: $\mathrm{CH}$ implies the undecidability of $\mathrm{CH} / \mathrm{NCH}$, but $\mathrm{NCH}$ does not imply that undecidability since cannot imply AC. All this is another argument in favor of QM and against LM-no-QM.

Anyway "QM \& an undecidability of QM/LM" satisfies almost all combinations of $\mathrm{AC}, \mathrm{CH}$, and their negations. Moreover it does not require LM-no-QM since LM and QM are complementary to each other where both AC \& $\mathrm{CH}$ hold.

As to the problem of "quantum gravity", this means the following: Quantum gravity as supposing QM is consistent as with $\mathrm{NCH}$ and the $\mathrm{AC} / \mathrm{NAC}$ invariance as with $\mathrm{CH} \& \mathrm{AC}$. However it is not consistent with $\mathrm{CH} \& \mathrm{NAC}$, in the domain of which general relativity is built, unfortunately.

What about LM-no-QM in "CN \& NAC"? Of course, one can construct QM on any LM there, too. That construction implies AC, and since NAC is valid there, the construction is forbidden, though. This is a very amazing state of affairs resembling the human rather than nature laws: QM is possible, but forbidden where general relativity is valid. After daring to construct QM in its territory, anyone turns out to be expulsed automatically in $\mathrm{CH} \& \mathrm{AC}$ where QM is admitted since it is complementary to LM and does not force LM to vanish.

What implies all that? Quantum gravity is a question of choice. One can create the theory as of quantum gravity as of general relativity, however ought to choose preliminary which of them. They should be equivalent to each other in a sense and can be thought as one and the same. Consequently general relativity can be reckoned as the cherished quantum gravity.

That is the case though it is very strange, even ridiculous. If and only if another and more general than QM measure be discovered so that the LM-no-QM be built constructively, then and only then general relativity and quantum gravity will be able to be distinguished effectively, i.e. experimentally. Vice versa: if an experimental refutation of general relativity be observed, a generalization of QM (GQM) will be implied: RIP both for Albert Einstein and for Niels Bohr since general relativity (LM) and quantum mechanics (QM) can be universal only together and reconciled. GQM will be able to resolve the dispute between them or will remove both when it comes. However we have not got any idea about GQM.

Finally, the example of BS can be used to illustrate how the strange kind of as if undecidability of $\mathrm{CH}$ to $\mathrm{AC}$, and hence the relation of general relativity and quantum mechanics in terms of measure:

BS implies CH according to the Alexandroff - Hausdorff theorem (Alexandroff 1916, Hausdorff 1916, cf. Sierpiński 1924): Any uncountable BS has a perfect subset (and any perfect set has the power of continuum). However, $\mathrm{CH}$ implies AC in turn, and the latter does Skolem's "paradox", i.e. the incomparability (or more exactly, unorderability) of any two infinite powers. Consequently, BS can be consistent as with $\mathrm{CH}$ as with $\mathrm{NCH}$ since $\mathrm{BS}$ and $\mathrm{CH}$ are complementary in a sense. If the case is $\mathrm{NCH}$, then $\mathrm{AC}$ is not implied and BS remains consistent 
as with $\mathrm{CH}$ as with $\mathrm{NCH}$.

Of course, this should be so as $\mathrm{BM}$ is a particular case of QM, and the latter is consistent with $\mathrm{NCH}$ (as well as $\mathrm{CH} \& \mathrm{AC}$ ).

All illustrate how it is possible for BS and BM to be consistent as with LM as with $\mathrm{QM}$ even where $\mathrm{CH} \& \mathrm{NAC}$ hold. That is the domain of general relativity, which should not exist if $\mathrm{CH}$ implies AC. Really $\mathrm{CH}$ implies AC only that $\mathrm{AC}$ implies the undecidabilty of $\mathrm{CH}$ or $\mathrm{NCH}$, which allows of existing the area of general relativity.

One can abstract the logical relation of general relativity and quantum mechanics by means of the same one of LM and QM. Roughly speaking, they are complementary because of a similar complementarity of $\mathrm{CH} / \mathrm{NCH}$ and $\mathrm{AC} / \mathrm{NAC}$ rooted in the amazing or even paradoxical properties of infinity: AC supposes a single infinity which ought to be countable. However, both $\mathrm{CH}$ and $\mathrm{NCH}$ suggest an infinite set of sets which can be countable $(\mathrm{CH})$ in turn, too.

That unordinary logical relation does not generate any contradictions. In fact, it contravenes only the prejudices. Anyway, we can attempt to explain and elucidate the reason of our confusion and misunderstanding:

Anything in our experience can be either an indivisible whole (a much) or divided in parts (a many): No "much" can be a "many" in the same moment and vice versa.

The above postulate is not valid as to infinity: It can be defined as that "much" which is a "many" or as that "many" which is "much".

Consequently it can be equally seen as a single "much" consisting of a "many" of parts (after AC) or as a many of indivisible wholes ("much"-s) (after $\mathrm{CH} / \mathrm{NCH}$ ).

To reconcile the two viewpoints onto infinity in a single illustration, one can utilize the image of cyclicality.

While anything else consists of something else and is not self-referential or cyclic, infinity is what consists just of it self-referentially or cyclically: Its "much" is forced to return back into it as many units.

AC suggests that cycle while $\mathrm{CH}$ or $\mathrm{NCH}$ unfold this cycle in a line. Consequently $\mathrm{AC}$ sees infinity as a well-ordering (line) bounded as a cycle while $\mathrm{CH}$ (or $\mathrm{NCH}$ ) as many cycles well-ordered in a line.

Any contradictions between them do not arise since both are the same seen from opposite perspectives.

\section{QM compared with $L M$ and with BM:}

QM can be compared as with BM as with the LM to stand out its essence and features:

\section{QM vs. BM:}

- Similarities:

- Both are supposed to be universal.

- Both generate probabilities where they are bounded.

- Both can be generated by BS.

- There is a common viewpoint, according to which QM can be considered as a 
three-dimensional or "tricolor" generalization of BM.

- Differences:

- QM is complete, BM is not.

- QM is three-dimensional, BM is one-dimensional.

- BM can be considered as the particular case where the three dimensions of QM coincides.

QM vs. LM:

- Similarities:

- Both are complete under NAC.

- QM and LM correspond to each other "two-to-two", i.e. " \pm to \pm " or in other symbols "square-to-square".

- No one of QM and LM can be deduced from the other or represented as a particular case of the other.

The differences from each other (see above) focus on a common 3D space where they vanish. One can utilize the metaphor of the two eyes or binocular sight for QM and LM.

- Differences:

dimensionality.

- QM is three-dimensional, while LM is of an arbitrary even infinite

- The dimensionality of QM does not correspond to that of the space measured, in general. They can be interpreted differently even in the particular case, where they coincide (three dimensions). The dimensionality of LM always coincides with that.

- QM is universal: It does not depend on the dimensionality of the space measured. LM is not universal: It does strictly correspond to the dimensionality of the space measured.

If one uses the metaphor of binocular sight for QM and LM, then their "global focus" is always in the "plane" of QM, while LM can represent the "local development or change" dimension by dimension.

\section{The origin of QM:}

QM arose for quantum mechanics when Heisenberg's (1925) matrix mechanics and Schrödinger's (1926a) wave mechanics were united by the latter one (Schrödinger 1926b).

Though Hilbert space guaranteed as a mathematical enough formalism, as John von Neumann (1932) showed in Mathematische Grundlagen der Quantenmechanik, for the quantum mechanics, the sense of that guarantee as well as its attitude toward the two initial components, matrix and wave mechanics accordingly, remained misunderstood:

Heisenberg's matrix mechanics represented all quantum motions only as discrete rather than continuous or smooth.

Schrödinger's wave mechanics represented all quantum motions only as smooth rather than discrete, though.

Consequently the sense of quantum mechanics, which unites both by means of Hilbert space, is that, in fact, all quantum motions are invariant to the transition between the discrete and smooth. 
However wave mechanics had advantage that it could represent that invariance in terms of the continuous and smooth, which terms were dominating for classical mechanics, though they were only prejudices, a legacy of the past, needless or even harmful:

The determent consisted in that the invariance of the discrete and smooth as to quantum motion remained tightly hidden in the mathematical apparatus of Hilbert space and accordingly misunderstood in physical interpretation.

The real sense of QM is to suggest a common measure both for the discrete and for the continuous and smooth so that to offer a suitable language for their invariance required by quantum mechanics.

The case of a (discrete) quantum leap measured by $Q M$ :

Any quantum leap can be decomposed in harmonics by Fourier transform:

Then any of those harmonics can be enumerated and considered as a QM for the $\mathrm{n}^{\text {th }}$ dimension of Hilbert space.

The $\mathrm{n}^{\text {th }}$ dimension of Hilbert space can be interpreted as a frequency or consequently, as an energy corresponding one-to-one to it.

The above construction shows the transition from real to complex Hilbert space and the transition from LM to QM as well. By the way the universality of QM is similar to that of complex numbers.

The case of a continuous or smooth physical motion measured by QM:

Since the continuous or smooth physical movement means a motion in Euclidean space, which is the usual three-dimensional one, it can be decomposed in successive 3D spheres or balls corresponding one-to-one as to all points of the trajectory in time as to the successive spheres or balls of the light cone in Minkowski space as well as to the successive dimensions of Hilbert space.

Consequently those points of the trajectory can be enumerated and considered as a QM for the $\mathrm{n}^{\text {th }}$ dimension of Hilbert space in an analogical way.

Now the $\mathrm{n}^{\text {th }}$ dimension of Hilbert space can be interpreted as a moment of time corresponding one-to-one to it.

The two above constructions show why QM is universal as well as the sense of that universality. Since frequency (energy) and time are reciprocal (or complementary in terms of quantum mechanics), then they can be juxtaposed as the two dual spaces of Hilbert space connected and mapped one-to-one by Fourier transform.

\section{Max Born's probabilistic mechanics:}

Max Born suggested in 1926 (Born 1926a, 1926b 1927a, 1927b; Born and Fock 1928; Born 1954) that the square of the modulus of wave function represents a probability, namely that of the state corresponding to that wave function. However somehow it was called the "statistical interpretation" of quantum mechanics. The term of "interpretation" used by Max Born himself as an expression of scientific modesty and politeness should not mislead. Its utilizing shows a complete misunderstanding of Max Born's conjecture and a yearning for its understatement. In fact it was not and is not an interpretation, but another, third form of quantum 
mechanics among and with matrix and wave mechanics. This is the cause for one to call it probabilistic mechanics (after the expressions of "wave mechanics" and "matrix mechanics" are common) rather than an interpretation.

Probabilistic mechanics shares Hilbert space with matrix and wave mechanics. However wave function (i.e. a point in Hilbert space) does not mean here a quantum leap decomposed in energies, neither a trajectory decomposed in time moments, but the characteristic function of a complex random quantity (or of two conjugate real quantity).

One should say a few words on the Fourier transform of a complex random quantity and on its characteristic function:

In fact their interrelation is quite symmetric and simple: The Fourier transform and the replacing of a complex random quantity by its conjugate swap the two dual Hilbert space.

Consequently the characteristic function of the conjugate of the complex random quantity is just the complex random quantity itself.

The interpretation of a complex random quantity and its conjugate is simple, too: Since a complex random quantity can be interpreted as two real conjugate (reciprocal) physical quantities such as e.g. time and frequency (energy), then the conjugate of the same random quantity must represent merely swapping between the corresponding physical quantities or the axes of the complex plane, or its rotation of $\pi / 2$.

Probabilistic vs. matrix mechanics: If one compares them, the differences would be only two: in interpretation and in choice between NAC and AC.

However the wave function in both cases and despite the differences would be the same and the same point in Hilbert space. That sameness inspires invariance as to probabilistic vs. matrix "interpretation" as to NAC vs. AC.

Since the wave function is a sum of the measured by QM, one can reduce completely that invariance in terms of QM:

QM as quantum probability guarantees the former members, and it decomposed in dimensions (which are harmonics or energetic levels in the case) supplies the latter ones.

A philosopher would emphasize the extraordinary universality both of Hilbert space and of QM contradicting common sense:

Why and where exactly? QM is so universal that can measure both the unordered (and even unorderable in principle) and the well-ordered and thereof ordering it (them):

In our case it can measure and order quantum probabilities (for the unorderable in principle) and quantum leaps (for the well-ordered in harmonics or energies), and therefore QM establishes a one-to-one mapping between quantum probabilities and quantum leaps:

That one-to-one mapping is too shocking to the prejudices. It shows that a level of energy corresponds exactly to a quantum probability: That is a physical quantity (what is the former) can be equated with a real number being without any physical dimensionality (what is the latter):

However this is what has been necessary for the objectives declared in the beginning of the paper: to demonstrate how QM allows of becoming nothing to something or vice versa and 
eo ipso creatio ex nihilo or reductio ad nihilum (i.e. true creation or true annihilation).

Probabilistic vs. wave mechanics: All what has been said above about the links between probabilistic and matrix mechanics can be almost literally repeated again in that case. The immaterial differences are as follows:

- The dual Hilbert space replaces its dual counterpart.

- The well-ordering in time replaces that in frequency (energy).

The one-to-one mapping based on QM establishes now a correspondence of a wave function as quantum probability with a continuous or smooth trajectory in time.

A threefold (even fourfold) one-to-one mapping arises thereof: It states invariance or equivalence in a sense between the quantum leaps (for the discrete), the smooth trajectories in time (for the continuous) and the quantum probabilities (for the unorderable in principle).

That threefold mapping shows how pure numbers even only the positive integers (for "nothing") can generate physical quantities in pairs of ("reciprocal") conjugates such as frequency (energy) and time. The stages of that generation are as follows:

- Nothing.

- The positive integers are given somehow, maybe by God as Leopold Kronecker reckoned: "Die ganzen Zahlen hat der liebe Gott gemacht, alles andere ist Menschenwerk" (cited in Weber 1893, p. 19):

- Creation: Qubits (or QM) replaces each of the positive integers generating Hilbert space.

- The Hilbert space generates that threefold mapping between quantum probability, energy and time and thereof the physical world arises already, too.

Quantum mechanics seen as the unification of all three kinds of mechanics: probabilistic \& matrix \& wave mechanics:

Quantum mechanics is better to be understood as the unification of all the three types of mechanics listed above instead only of the last two.

The sense of that unification is the extraordinary invariance (or equivalence in a sense) of the discrete, continuous (smooth) and the probabilistic in the common form of quantum motion:

Quantum motion can be already thought as a relation between two or more states despite whether each of them is considered as a discrete, continuous (smooth) or probabilistic one since it is always represented by one the same wave function in all the three cases:

This calls for far-reaching philosophical conclusions, though:

The difference not only between the discrete and continuous (smooth), but also that between both and the probabilistic is only seeming and accidental or even anthropomorphic in a sense.

Quantum motion breaks down their barriers and allows any transitions between them.

The probabilistic can be located "between" the discrete and continuous (smooth) and can be considered as something like a substance of that kind of transition. Accordingly the discrete and continuous can be supposed as the two extreme or particular cases of the 
probabilistic, which are opposite to each other, and that is not all:

What is the physically existing according to common sense can be linked only to those two extremes. Physical reality ostensibly consists just of (and in) both since they are all the actual.

According to the same common sense the probabilistic cannot be physically real since is not actual: However quantum mechanics shows that is the case, the probabilistic is physically real: "So much the worse for quantum mechanics because this means that it is incorrect or at least incomplete", declared the common sense then. Quantum mechanics rather than that "common sense" turns out to be the right again, though, experimentally verified (Bell 1964; Clauser and Horne 1974; Aspect, Grangier, and Roger 1981, 1982, etc.).

If quantum mechanics is the right, what does it mean about the philosophical interrelation between reality and "virtuality"?

"Virtuality" is a term coined here to denote just that new class required by quantum mechanics and involving both reality, i.e. the discrete and continuous (smooth), and "only" (ostensibly) the possible so that to allow of any transfer between them.

Consequently virtuality is a term for the new constitution of being, according to which the barriers between the actual and the possible are broke down and all the kind of transitions between them are unrestrained.

Thus virtuiality established by quantum mechanics can resolve our properly philosophical (and even theological) problem about creatio ex nihilo or reductio ad nihilum: The area of probability can describe very well both those creatio and reductio as states and processes: One can see the actual in creating or annihilating rigorously mathematically, i.e. in the process of creation or annihilation, as the change of probability.

Not less striking is that the new "constitution" of virtuality suggests for mathematics to be more general than physics if the latter is defined and restricted only to the actual; or in other words, mathematics and a new and more general physics can and even should coincide.

How to interpret the fermion and boson kind of spin statistics in the light of that unification?

According to the so-called spin-statistics theorem (Fiertz 1939; Pauli 1940) all the quantum particles can be divided into two disjunctive classes after the second quantization: fermions and bosons:

Since the second quantization maps the wave functions of the quantum particles "two-to-two", it admits two kinds of solving as to a swap of the space-time positions of two quantum particles: symmetric $(++,--)$ and antisymmetric $(+-,-+)$.

The bosons are supposed to be those of symmetric swap, and the fermions are those of the antisymmetric swap. Turns out the any number of bosons can share one the same state and wave function while if they are fermions, only two.

The following can be easily spotted: Quantum probabilistic mechanics explains very well that property as to the bosons, and quantum matrix-wave mechanics explains it not less successfully as to the fermions: 
Indeed the opportunity of sharing a common state or wave function by the bosons is due to the sharing of a common probability by an arbitrary ensemble of quantum particles. That ensemble, which possibly consists of an infinite number of elements, is supposed not to be well-ordered.

The same ensemble already well-ordered can be distinguished in two kinds of well-ordering corresponding to the two fermions admitted in one the same state or wave function. The one is well-ordered to, and the other from infinity. If the ordering is in time and energy, the one fermion as if corresponds to the discrete "half" of wave-particle duality, and the other accordingly to its continuous (smooth) "half".

Hence one can clearly see that the second quantization giving rise to spin statistics either is equivalent to, or is a particular case of that quantum mechanics, which includes as probabilistic as matrix and wave mechanics. Indeed, the sense of the second quantization is to be defined "quantum field". In fact, this is done by ascribing a wave function (i.e. a quantum state) to each space-time point. That quantum mechanics, which includes as probabilistic as matrix and wave mechanics, ascribes a space-time point to each wave function. Then:

If the quantum field is well-ordered, then the mapping between all the wave functions (Hilbert space) and all space-time points (Minkowski space) is one-to-one, and the second quantization is equivalent to that quantum mechanics, which involves probabilistic mechanics. Then any quantum particle must necessarily be either a boson or a fermion.

If the quantum field is not well-ordered, it admits two opposite options as well as both together:

Two or more space-time points to share one the same wave function, and thus the reverse mapping not to be well-defined: It will not be a standard function.

Two or more wave functions to share one the same space-time point, and thus the straight mapping not to be well-defined: It will not be a standard function.

However the most general case is two or more space-time points to share two or more wave functions (i.e. together both above). If that is the case, it can be equally described as some space-time points, which share a part of some wave functions (entanglement) or as wave functions, which share a "part" of some space-time points ("quantum" gravity). The interrelation or equivalence of entanglement and gravity is being studied.

If the quantum field is not well-ordered as above, it can be represented in a few ways (as well as in their combinations or mappings):

- As a curving of Hilbert to Banach space.

- As a curving of Minkowski to pseudo-Riemainian space.

- As quantum particles with an arbitrary spin: such which can be any real number.

The transitions between the "probabilistic" wave function and "well-ordered" wave function in any of the above ways describe in essence the arising of "something from nothing and from time" ("time" is for the axiom of choice) as a continuous process as a quantum leap as well as a purely informational event.

One can give examples of that arising in terms of classical (gravity) or quantum 
(entanglement) physics as a continuous process.

Quantum mechanics, $Q M$ and $A C$ :

A question may have remained obscure: Does quantum mechanics need AC?

Quantum mechanics is actually the only experimental science, which requires necessarily AC: Roughly speaking, the state before measuring has not to be well-ordered, but after that it has to. This means that measurement supposes the well-ordering theorem, which is equivalent to AC:

The "no hidden variables" theorems do not allow of well-ordering before measuring.

However even only the record of the measured results (which is after measuring, of course) forces they to be well-ordered.

The basic epistemological postulates equate the states before and after measurement and thus imply AC.

Though measuring requires the $\mathrm{AC}$, it remains inapplicable before measuring. Consequently quantum mechanics is ought to be consistent both with $\mathrm{AC}$ and NAC in addition.

The only possible conclusion is too extraordinary: Quantum mechanics is consistent as with $\mathrm{AC}$ as with NAC. However, quantum mechanics is not consistent with the absence as of $\mathrm{AC}$ as of NAC.

We could see above that QM is linked to AC in the same extraordinary way. This means that quantum mechanics is consistent with $\mathrm{QM}$ as to $\mathrm{AC}$, which should expect.

Quantum mechanics, $Q M$ and the $C H$ :

That extraordinary interrelation between quantum mechanics, QM and AC goes on with $\mathrm{CH}$ :

$\mathrm{NCH}$ is consistent as with $\mathrm{AC}$ as with $\mathrm{NAC}$, thus quantum mechanics is consistent with $\mathrm{NCH}$.

Reversely, $\mathrm{CH}$ should (ostensibly) imply AC. However AC implies the undecidability of $\mathrm{CH}$ and $\mathrm{NCH}$, then $\mathrm{CH}$ implies by means of $\mathrm{AC}$ the own undecidability. The only way out is then to admit the complementarity of $\mathrm{CH}$ and $\mathrm{AC}$.

Both quantum mechanics and QM share that extraordinary relation to $\mathrm{CH}$ by means of AC.

Though the state of affairs is strange, it is not logically contradictory. It messes up only common sense. The cause of that ostensible muddle is the intervention of infinity, of which we try to think as of a finite entity.

\section{Physical quantity measured by QM or by LM, or by BM:}

The definition of physical quantity in quantum mechanics involves measuring by QM. It is a generalization of the corresponding notion in classical physics and exact science.

One can see the quantum at all as that generalization from LM and BM to QM. The correspondence is the following: 
Table 1: From LM and BM to QM

\begin{tabular}{|c|c|c|c|}
\hline & Quantity & Unit & Value \\
\hline The classical case & Real quantity & Unit & Real number \\
\hline The quantum case & Wave function & Conjugate wave function & Self-adjoint operator \\
\hline
\end{tabular}

A few conclusions can be drawn from that correspondence:

The sense of a point of dual Hilbert space is to be a "unit", or something like a reference frame, which can measure a point of Hilbert space.

The measured value represents the distance between the "unit" point (or its conjugate point, too) and another point, which is for the measured quantity. This distance can be thought of also as a distance in the "reference frame" of that point.

Both cases are "flat": They conserve the measure under translation and rotation. If the translation and rotation are understood as usual as translation and rotation in space-time, then that "flatness" implies the classical laws of conservation complemented by Lorentz invariance. One can especially emphasize the time translation and energy conservation.

The "flatness" in general can be equated with the axiom of choice. Indeed the well-ordering requires that flatness since otherwise a second dimension for ordering appears questioning the well-ordering made only in the first dimension.

The above table (1) can be paraphrased in terms of the "crooked" as the following table 2 asking how both tables can refer to each other:

Table 2: Table 1 paraphrased

\begin{tabular}{|c|c|c|c|}
\hline & Quantity & Unit & Value \\
\hline The classical case & Real quantity & Unit & Real number \\
\hline The "crooked" case & Contravariant vector & Covariant vector & Metric tensor \\
\hline
\end{tabular}

The "crooked" case is that of general relativity and gravity. The question for the connection between the two tables addresses the problems of quantum gravity in terms of general relativity and measure theory.

To be together in front of the eyes, one can combine the two tables as a new one (3):

Table 3: Table 1 and Table 2 unified

\begin{tabular}{|c|c|c|c|}
\hline & Quantity & Unit & Value \\
\hline The classical case & Real quantity & Unit & Real number \\
\hline The quantum case & Wave function & Conjugate wave function & Self-adjoint operator \\
\hline The gravity case & Contravariant vector & Covariant vector & Metric tensor \\
\hline Quantum gravity & $? ? ?$ & $? ? ?$ & $? ? ?$ \\
\hline
\end{tabular}

The above table (3) shows that the problem of quantum gravity is a problem of measure: It concerns the ostensibly contradictory properties of infinity focused on how AC and $\mathrm{CH}$ should refer to each other. 
The gravity being as "crooked" as smooth in general relativity supposes the "classical" case of NAC and $\mathrm{CH}$. However as $\mathrm{CH}$ implies AC, it should not exist. After all it arises anyway since AC in turn implies the undecidability between HC and NHC. In last analysis it concerns the property of infinity to be both cyclic and linear unlike anything in our usual experience.

One can think of the quantum and gravity case as complementary. In particular this means that the values of a quantity as a self-adjoint operator or as a metric tensor are complementary, too, as well as QM and the "crooked" LM therefore that QM and LM are even equivalent in the distinctive way of quantum mechanics

Just this complementarity of QM and LM is taken in account as to $\mathrm{NCH}$, which is consistent as with AC as with NAC, and for this, with the curious invariance of AC and NAC.

For the above three as to the $\mathrm{CH}$ case, one can be free to suggest that it exactly repeats the $\mathrm{NCH}$ case in relation to $\mathrm{AC}$ and NAC because of the undecidability between $\mathrm{CH}$ and $\mathrm{NCH}$ after AC. This would means that any theory of quantum gravity is not necessary since the pair of quantum mechanics and general relativity can represent whatever the case is.

The following should be highlighted in background of the just said: In the same extent, one can be free to admit the opposite: That is the $\mathrm{CH}$ case does not repeat the $\mathrm{NCH}$ one just because of the used undecidability between $\mathrm{CH}$ and $\mathrm{NCH}$ after AC. This will say that a theory of quantum gravity is possible though it will be never necessary.

One can compare with the real state of affairs: Indeed many theories of quantum gravity appear constantly and supposedly some of them do not contradict the experiments just because they are possible. However they are not necessary in principle since general relativity does not contradict the experiments, too, and "Occam's razor" removes all of them remaining in hand only general relativity.

\section{QM and quantum quantity in terms of the Bekenstein bound:}

The Bekenstein $(1972,2005)$ bound determines the top limit of the entropy in a given volume containing a given energy. Bekenstein himself interprets it as the quantum bridge between the two kinds of entropies: the mathematical (informational) and the physical (thermodynamic) one (Bekenstein 2003).

Given the Bekenstein bound and the laws of thermodynamics, general relativity can be deduced from them (Jacobson 1995; Smolin 2002 173-175).

After all said one can accepted it as the missing quantitative link between the wave function in "probabilistic mechanics" and it in matrix-wave mechanics, more exactly between the change of the former and that of the latter. Indeed:

If the case is the "flat" one, the two changes have to be equal, and the maximum allowable limit of the Bekenstein bound is reached. Both changes represent some quantum quantity (i.e. measured by QM) in the same way despite whether it is of a boson or of a fermion. That is the "flat" case of quantum mechanics.

The ratio of the "two entropies" corresponds to temperature as to the classical case where both are real function accepting consequently only real values. More exactly the 
temperature can be thought as the ratio of the physical entropy (in units of energy) and the mathematical one (dimensionless).

Utilizing the Bekenstein bound, one can introduce a new quantity of "quantum temperature", which will generalize the "classical" temperature, though it is a real function:

Thus the ratio of the "two entropies" corresponds to quantum temperature as to the quantum case despite that both are real functions accepting consequently only real values. More exactly the quantum temperature can be thought as the ratio of the physical entropy (in units of action) and the mathematical one (dimensionless).

One can notice that the notion of quantum temperature expresses the rate of "crooking" and hence, gravity. Indeed:

The quantum physical entropy (i.e. the top limit itself) represents the "flat" case as if as a reference frame for measuring the rate of "crooking", or as a zero level of it. Consequently:

There is a zero level of quantum temperature expressing the absence of gravity and corresponding to the zero level of temperature ("absolute zero of temperature") being its generalization:

About the way and sense of that generalization: If translation in time and thus energy conservation is valid, then the mapping between the temperature and the quantum one is one-to-one. The notion of quantum temperature is redundant if that is the case and Occam's razor would remove it.

Therefore the way of generalization is to correct the time translation as well as energy conservation by a coefficient in depend on the ratio of "crooking": Gravitational energy is that correction in energy conservation in depend on the "crooking".

The correction of gravitational energy is added while the correction by means of the ratio of crooking has to be multiplied. So the former has to depend on time (distance) while the latter has not to. The former is local and in space-time while the latter is global and can be thought as out of space-time.

The sense of generalization is that: to avoid the restriction imposed by energy conservation just as general relativity does the same though in another way.

If the quantum temperature and gravitational energy are so closely connected, then whether the usual defined temperature and gravitational energy are not linked by an exact mathematical formula, too:

No, they are not connected by any formula since they are independent of each other.

Indeed, the condition of that transition from quantum to standard temperature is the "flatness" in question. After this is the case, then translation in time holds as well as energy conservation: Thus gravitational energy has to be zero since it does not keep in general. Reversely, is there any nonzero gravitational energy, the quantum temperature cannot be reduced to the standard one.

In fact, quantum temperature can be represented as a function of two independent variables: a newly defined temperature of gravitational energy and standard temperature. The former is the ratio of the gravitational energy divided by the entropy of its space-time 
distribution. However if the case is that of general relativity postulating the equality of gravitational and inertial mass, first, gravitational energy has to be equated with mechanical energy, and then the newly defined gravitational and standard temperature must coincide entirely. Though quantum temperature can be defined being a function of two ostensibly independent variables, it does not make much sense since they are accepted equal as an axiom.

QM is reduced to two orthogonal BM or LM, which then are postulated forever equal. In fact quantum mechanics tolerates such resolving in its own territory since complementarity as forbids as admits any ratio between the orthogonal components of QM (which both are BM or one-dimensional LM). The Occam razor will remain then the ratio of unit, since this is the simplest suggestion, i.e. general relativity for any quantum gravity.

While quantum temperature is a real quantity and function, the above ratio is purely hypothetical: General relativity wins against any quantum rival.

One can give an illustration for quantum temperature in term of the standard one utilizing the notion of the Gibbs and the Boltzmann entropy in an adiabatic process: The ratio of the Gibbs to the Boltzmann entropy is that illustration of quantum temperature. Then an adiabatic heating would be possible after decreasing the Gibbs entropy.

One must expressly emphasize that quantum temperature can be defined only under condition of quantum correlations rather than classical ones. So the above is only an illustration but not an example of quantum temperature. The correlations which can distinguish the Gibbs from Boltzmann entropy could be both quantum and classical anyway. However given a one-to-one mapping between quantum and standard temperature, there can be no quantum correlations after all.

What is the necessary and sufficient condition for quantum correlations is just QM. Two or more variables independent of each other by classical correlations none the less can be dependent on each other by quantum correlations. This shows the EPR "paradox" (Einstein, Podolsky, Rosen 1935), which forecast the phenomena of entanglement studied by quantum information, though in the form of an alleged "incompleteness of quantum mechanics".

The quantum correlations in question appear for the two additional dimensions in QM in comparison with the single one of BM. The same refers to quantum and classical probability. As to the terms of set theory, it requires the invariance to AC or Skolem's "relativity" (1923):

Given BM, two or more variables can correlate if and only if the sets of their values have a nonempty intersection. The hypothesis that the same holds in quantum mechanics wears the name "hidden variables". However given QM, a "clever backdoor" appears to suspend the above: There can be correlations only before any well-ordering so that the intersection of the corresponding sets obtained after the well-ordering as the result of measuring is empty, though: If this is the case, there are quantum correlations but there are no classical ones.

The next step in the pathway of generalization is that to pass from quantum temperature, which is a real function, to a complex quantum temperature in an appropriate way:

In general case one has to introduce a "quadrangle". The four vertices of that "quadrilateral" will be two energies and two entropies. A virtue of the metaphor is that all the 
particular cases of a quadrangle (square, rhombus, rectangular, trapezoid, isosceles trapezoid) can make visual the analogical particular cases in the area of quantum gravity.

- Mechanical energy ("vertex" A);

- Gravitational energy (B);

- The entropy of the space-time distribution of mechanical energy (C);

- The entropy of the space-time distribution of gravitational energy (D).

The "sides" are as follows:

"AB": If "A", mechanical energy, and "B", gravitational energy coincide, this is equivalent to equating for gravitational and inertial mass, i.e. Einstein's "Äquivalentzpirinzip" (1918). Consequently, the "length" of AB is a measure of another energy, which has to be in a sense a "difference" between mechanical and gravitational energy, therefore perhaps a corresponding mass, too.

Anyway if that "difference" has energy but not mass, thereof violating "Machshes Prinzip" (Einstein 1918), this would be yet possible and would mean that the "velocity" of the interaction, which corresponds to that nonzero energy, has to be infinite or out of time in other words. All phenomena of entanglement would satisfy that condition.

"CD": If the length of CD is zero, this implies that the two conjugate distributions coincide, i.e. those are in fact one the same real distribution. Thereof that "length" should signify what is the ratio or subtraction between the phases of the distributions: an infinite-dimensional phase, too, in general. In other words, this means that "CD" is entanglement: more exactly the entanglement of all parts of a quantum system, and its "length" is the degree of entanglement.

If the " $C$ " and "D" coincide, this implies in final analysis that Einstein's (1918) "Relativitätsprinzip" is satisfied, i.e. all physical phenomena are "zeiträumliche Koinzidenzen", and consequently space-time is universal: Nothing physical is out of space-time.

So, one can assure that all the three Einstein (1918) principles of general relativity as he himself formulated them applying together force the "quadrangle" ABCD to degenerate to a single point, and its "area" to be zero.

Reversely, the same shows that an arbitrary quadrilateral such as that of consideration, "ABCD" has to be a generalization of general relativity, perhaps even the cherished quantum gravity:

In particular, if and only if one has abandon general relativity, the two following temperatures being the corresponding components of complex quantum temperature make sense to be considered:

"AC": It can be called time temperature (or space-time temperature). To continue the analogy with the standard temperature, its measure will be logarithmic, too, so that the zero degree of entanglement maps one-to-one as the zero level of time temperature: It will be the absolute zero of time temperature: So something like "anti-entanglement" is not known at least at present, as the negative values for the time temperature will be forbidden.

The physical sense of time temperature is the degree of space-time expanding. Consequently, it should be connected with the process of space-time genesis. However that 
process or genesis is not in the time, but of the time in a sense:

One can penetrate that process in a few ways:

Mathematically, this is the process of well-ordering in time or in space-time. It flows for or in the increasing of space-time entropy or in other words, together with the decreasing of entanglement degree.

However physically, the increasing of entropy in a system corresponds to time.

The mathematical and physical way to think of the links between time and entropy can be united as a kind of mapping between them: The one of them corresponds to the straight mapping, the other to the reverse mapping.

Time temperature can refer to the universe as a whole. Then its decreasing or change describes the expansion or change of the universe.

Time temperature can refer to any quantum particle separately as well. Then the decreasing or change describes the emancipation of the particle in question from its environment, i.e. the process of its genesis, the way for it to appear.

Consequently, the time temperature allows of thinking of both the cases (as the universe as a quantum particle) uniformly.

"BD": It can be called energy temperature (or energy-momentum temperature). The same analogy with standard temperature should expand over energy temperature and thereof all about the absolute zero of energy temperature and forbidding its negative values is implied.

The physical sense of energy temperature would be analogical to that of time temperature.

The distinction between time temperature and energy temperature makes sense to be elucidated:

Time temperature and energy temperature are directed oppositely. Time temperature decreases while energy temperature increases.

The increasing of energy temperature describes the process of how anything acquires its mass. Consequently space-time expansion (i.e. the decreasing of time temperature) and energy (mass) increasing (i.e. the increasing of energy temperature) have to go hand in hand right what one observes as to the universe.

The conception of the "Big Bang" can be reinterpreted since it supposes energy conservation forcing to search some different source for the "dark" energy and mass constantly springing somehow.

So the notion of quantum temperature has to describe how time temperature turns into energy one, or in other words how anything including the universe itself can arise and constantly arises from time and from nothing without any other cause of this.

The quantum "flattening" vs. the general-relativity "equating" as to mechanical movement:

Both quantum mechanics and general relativity consider and juxtapose two types of quantities to describe mechanical movement: They refer correspondingly to space-time and energy-momentum. According to quantum mechanics, they are conjugate to each other and are 
incommeasurable together. As to general relativity they are commeasurable though the relation between their measures is represented by metric tensor in any space-time point and it is what is responsible for the space-time curvature and for gravity in final analysis.

In both cases there are two measures, which are as different as equally valid, and have to be combined and superposed with each other somehow. This "somehow" in turn is different and even incombinable between the two cases: that of quantum mechanics and that of general relativity

The solution of quantum mechanics consists of two consistent steps:

The first one is to forbid for them to be considered together if they are two one-dimensional measures, i.e. BM or LM.

The second one is to introduce QM, which is able to unite them as two different (and "orthogonal") particular cases of its.

That construction shows that the measure utilized by quantum mechanics is inconsistent with that of general relativity.

Anyway they can be equated in a sense if the second step introducing QM be delayed and two pairs of two one-dimensional measures are what is juxtaposed.

The first pair, which will be considered, is that according to quantum mechanics. Since the two members of it are incommeasurable, one is free to postulate conventionally any ratio between them, and let it be one as the simplest case.

The second pair is that of general relativity. Since the ratio of its member exists and it is an arbitrary real number, then that state of affairs can be equivalently expresses by introducing a corresponding angle between the two measures:

What should be the ratio of the units of the two measures. In fact there is no theoretical reason to answer it. As in the case of quantum mechanics, the simplest suggestion could accept, too, and it is the same: their ratio to be one.

However unlike the case of quantum mechanics, general relativity can found it on a firmly established fact: the equation of gravitational and inertial mass: It means the equation of the corresponding energies, which in tradition are called potential energy connected with a space-time point and kinetic energy referred to an energy-momentum point.

If and as far as quantum mechanics acclaims energy conservation, as Pauli did unlike Bohr, Kramers, and Slater (1924), then it accepts in fact implicitly the same ratio of one unit as general relativity though complementarity allows of that ratio to be any real number. Indeed the two members of the energy-conservation equation are incommeasurable together. These are potential and kinetic energy in question, which depend correspondingly on position and on momentum.

One can compare the ways of quantum mechanics and general relativity for the two measures to be reconciled with each other:

Quantum mechanics says: The two measures are incommeasurable, but they can be equated conventionally and in tradition.

General relativity says: The two measures are angled arbitrarily, for which space-time 
is curved, and that curvature represents gravity for us. The ratio between their units is one unit according to the equation of inertial and gravitational mass, though.

The above statements are similar and moreover that of general relativity is stronger, it implies that of quantum mechanics. More exactly, the latter requires the measure to be equal if and only if they are "flattened" while the former insists on their equation even if they are arbitrarily angled.

However given the allegation of quantum mechanics, it implies the negation of its premise, that of general relativity, since the former does not admit at least the notion of angle between the two measures being incommeasurable.

One can see oneself forced to justify and elucidate a specially "quantum" logical rule, which generates an extravagant kind of mutual independence between any two propositions satisfying the following two conditions:

(A implies B) implies that there exists B so that: (A implies B) \& (B implies C).

Let $B$ be not-A (It is possible for the "paradox" of material implication). Since not-A is equivalent with ( $\mathrm{A}$ implies not- $\mathrm{A}$ ), then the not- $\mathrm{A}$ by $\mathrm{B}$ being not- $\mathrm{A}$ implies that $\mathrm{A}$ implies not-A, and thus the existence of B.

What is the term of the relation between $\mathrm{A}$ and $\mathrm{B}$ or of those $\mathrm{A}$ and $\mathrm{B}$ if they relate to one another so? For the absence of a term it can be called now complementary independence of two statements: Though the one of them implies the other and seems initially to be dependent on each other, there turns out anyway that the latter statement in turn implies the negation of the former one. Both the propositions satisfying those conditions are consistent with each other and a few examples of similar pairs of propositions were enumerated already above.

That kind of "complementary independence" can be commented so: B exists necessarily and only "purely" in such a sense: The former condition requires for B to exist, but the latter one forbids any effective construction for the empty intersection of A and not-A. This is reminiscent of the "clever backdoor", through which quantum correlations can sneak behind the classical ones: In other words, one can consider the classical correlations as constructive, and ascribe only pure existence as to the quantum ones.

That complementary independence is absolutely enough. Having large experience with complementarily independent propositions as above, quantum mechanics can offer a few useful logical ideas:

- Infinite constructive procedures or algorithms can be discussed in a finite way.

- That discussion requires not only the axiom of choice but also the equivalence (or invariance, or relativity) in relation to it.

- A proposition of "pure" existence corresponds to any infinite procedure or algorithms.

However given such a proposition, two infinite procedures or algorithms, which can coincide in particular, correspond to it. This is so for the infinite constructive procedure or algorithm admits in principle two directions, which can be conventionally designated as "to infinity", and "from infinity". 
The relation between corresponding infinite procedures or algorithms, on the one hand, and the propositions of pure existence, on the other, is that of complementary independence.

Another question is relevant to how general relativity refers to quantum mechanics: It is about the cosmological constant introduced in the equations of general relativity later and which Einstein himself called his "biggest blunder" (cited in Gamov 1970 p. 44) after Hubble's discovery that the universe expands.

One can start by elucidating the sense of the cosmological constant in terms of QM:

In fact a nonzero cosmological constant revises the equation of inertial and gravitational mass and thereof the equation of the two measures, e.g. those of potential and kinetic energy, too. Though the revision, the cosmological constant can remain consistent with the equation of inertial and gravitational mass in the following way:

The equation of inertial and gravitational mass holds locally while the nonzero cosmological constant is global. In fact this would lead to the quadrangle ABCD considered above in final analysis but with a difference in interpretation: The side CD corresponding to probabilistic mechanics is interpreted as the representation of the global, i.e. as that of the system as a whole, which is the universe as a whole. One can notice that Einstein's introducing of the thermodynamic properties of the system as a whole (Einstein 1910, pp. 1276-1282) corresponds to the other idea about the cosmological constant expressing an "emancipation" of the whole analogical in both cases. By the way, Einstein explains there the well-ordering of all states in time so: A single state possessing a corresponding probability overwhelming those of all adjoining states follows any state practically always (ibid. 1277) ${ }^{1}$. This is not so in the quantum case: However the apparatus plays the role to enhance the difference in probability between all the possible adjoining states and thus to choose only one as a single one implied by the well-ordering in time.

The cosmological constant can be generalized as a function of space-time or of other variables. Then locally, it would delineate the same as the gravity in general relativity does by means of the curvature of space-time: It is absolutely redundant locally, because of which its sense can be related only to the global.

However as to the bridge between quantum mechanics and general relativity, the cosmological constant is able to make sense locally as the forbidden, inexplicable or arbitrary difference between the two dual spaces or measures.

In terms of QM, the cosmological constant could represent the difference between QM and any two one-dimensional LM or BM arbitrarily angled, though.

In return to the sense of the cosmological constant, gravity can be interpreted as an equivalent expression for the above difference between QM and any two one-dimensional LM or BM arbitrarily angled.

${ }^{1}$ Cf. Born and Fock (1928); Avron and Elgart (1999). 
Here are the considerations of Einstein himself to introduce the cosmological constant in his paper (Einstein 1918):

The initial equation of general relativity, which is without the cosmological constant, admits the gravitational field in the absolute vacuum, i.e. even at zero energy.

To prevent such an option, which seemed to him to be odd, he introduced "Machsches Prinzip", according to which the only possible source of gravitational field can be the mass (correspondingly energy).

In fact, that principle is imposed to be added for the suspension of Lorentz invariance in general relativity: If it is absent, the velocity can be infinite and thus the entities, which have the zero relativistic mass (not the mass at rest), are able to possess nonzero energy and to generate a gravitational field. In other words, the absolute vacuum can have got energy (but not any mass) and a corresponding gravitational field. To Einstein that idea seemed absurd, but not to Dirac who introduced implicitly the notion of "vacuum energy" as a sea of negative energy (Dirac 1931) since his relativistic (and for that Lorentz invariant) generalization (Dirac 1928, 1930) of the Schrödinger equation implies its existence.

After quantum information and all phenomena of entanglement, the idea does not seem absurd on other, quite different and completely independent grounds. For its infinite velocity, the entanglement can possess nonzero energy but zero relativistic mass.

What could possess energy but not mass as above (eo ipso violating the Mach'shes prinzip) is quantum information as a substance or an element of the world and universe. Such an opportunity arises in final analysis for QM, but remains impossible in framework of LM or BM. Since the Einstein equation (without the cosmological constant) admits it, the sense of the cosmological constant can be interpreted as an expression or pure sign for "complementary independence": Then the exact value or function of the cosmological constant should remain forever inexplicable in principle.

The interpretation in terms of the "quadrangle ABCD" above is not less interesting: The first two Einstein principles ("Relativitätprinzip" and "Äquivalenzprinzip") cannot guarantee the perfect degeneration of that quadrangle to a point but only to a segment " $\mathrm{AC} \equiv \mathrm{BD}$ " $(\mathrm{A} \equiv \mathrm{B}$ for "Relativitätprinzip", and C $\equiv \mathrm{D}$ for "Äquivalenzprinzip"). Consequently, the "Machsches Prinzip" was called to remove that last imperfection in the degeneration.

The interpretation in terms of quantum temperature is: The "Machsches Prinzip" is equivalent to the zero quantum temperature designating the lack of any entanglement (or the zero entanglement). Reversely, after the quantum temperature has been already introduced, this inevitably means for it to be suspended or on other words, generalized first as a nonnegative real, and then as a complex number. Consequently, the notion of quantum temperature is consistent with the initial Einstein equation without the cosmological constant as well as with its generalizations where the cosmological constant is a real or complex function.

However Einstein himself declared the cosmological constant for the "biggest blunder" of his (cited in Gamov 1970, p. 44), to see why:

Though Einstein founded the cosmological constant in 1918 (the quoted paper) by the 
newly "Machsches Prinzip", the real reason to introduce it in a paper a year earlier (Einstein 1917) was to conserve the universe static since the equation without the cosmological constant implied that it should expand.

Hubble invited (1931) Einstein himself to assure that the universe is expanding. It was Hubble's discovery that made Einstein to change his mind and to declare his "biggest blunder". Really if he had remained the equation as it had been initially (i.e. without the cosmological constant), then his scientific genius would obtain a new magnificent acknowledgement for an amazing forecast.

In fact the expanding universe should reject the "Machsches Prinzip", too, since it was introduced just to conserve the universe static. This means that there exists another possible and even really acting source of gravity different from the mass, i.e. that source must not possess any relativistic mass, but nevertheless it has to have some nonzero energy, because it acts.

The "Big Bang" is the first conjecture for that mysterious source of energy. Its virtue is for energy conservation to survive (in fact, almost). About 13 or 14 billion years before, all the energy of the entire universe appeared from the nothing in a single instant "according to God's will" and without any natural cause, but energy conservation holds after that abominable crime on it committed by the "Big Bang".

However if the "Machsches Prinzip" is rejected, then the segment " $A B \equiv C D$ " above cannot degenerate to a point, and one can search in it a constantly acting natural cause violating energy conservation. Its time integral is depicted back as the "Big Bang".

If one accept the length of the segment " $\mathrm{AB} \equiv \mathrm{CD}$ " as the quantitative degree for the violation of the "Mach'sches Prinzip", it would correspond one-to-one to the degree of entanglement or to the quantity of quantum temperature (as a real quantity).

The hypothesis is that entanglement and only entanglement is the single source both of the violation of the "Mach'sches Prinzip" and of the corresponding energy. As Einstein introduced that principle to found the static universe, then on the contrary, the fact of the expanding universe can direct to entanglement as the source of the energy, which is usually and perhaps groundlessly ascribed to the "Big Bang".

The existence of the universe suggests a huge violation of energy conservation, which can be explained in two alternative and possibly complementary ways: as an enormous energy leap, the Big Bang in question, as well as a constantly acting cause, the entanglement. This is the quantity of quantum temperature, which allows of them to be equated. So the "Big Bang" explanation is partial and traditional rather than untrue or incorrect. However the violation of energy conservation is absolutely necessarily in both cases. The entanglement explanation has advantage to point a natural and constantly acting cause, but it is not less partial and one-sided than the "Big Bang" one. The notion of quantum temperature involves implicitly an equivalence (or invariance to, or relativity) of those two kinds of explanation.

If the quantum temperature is involved as a complex rather than real quantity, then the violation only of the "Mach'sches Prinzip" is not yet enough: It already requires the violation of at least one of the rest two principles: Einstein's “Äquivalentzpirinzip" or Einstein's 
"Relativitätsprinzip", so that the real and imaginary component of quantum temperature are able to be distinguish from each other.

However, general relativity suggests a description absolutely equivalent to that by means of quantum temperature as to the two unmatched components of its. This is just gravity which represents their mismatch as a force, namely that of gravity or as a curvature between the two components under the condition of their coincidence. Consequently the two quoted above Einstein principles should be understood as convention for the form of description rather than as natural laws.

QM can be compared with BM and LM in terms of general relativity and its curved space-time as follows:

QM is a universal three-dimensional measure: Its three dimensions admit another interpretation: as the measures of two conjugate one-dimensional physical quantities and a third one for the "curvature" between them, addressing the measure conception of general relativity.

There exists a difference between them. The three dimensions of QM are absolutely equivalent and interchangeable: They are as isotropic as the three dimension of Euclidean space. Unlike this, the corresponding triple of measures in general relativity are not equivalent to each other neither interchangeable. Being differently nominated, they consists a well-ordered triple, which is anisotropic.

There exists a common essence rather than a mere analogy between anisotropy, well-ordering (thus the axiom of choice), and nominating, on the one hand, and also between isotropy, the lack in principle both of well-ordering (which means the negation of the axiom of choice) and of any nominating, on the other hand. This allows the invariance to the axiom of choice to be applied to finite sets as well. It establishes an equivalence between the elements of any set if they are nominated (enumerated) or not. If the dimensions of a space are considered as that set, that invariance between isotropy and anisotropy can be discussed.

The term of "complementary independence" has been introduced above just to describe the same kind of logical relation between QM and LM (BM). One can utilize it as for the finite invariance to the axiom of choice as for the infinite one above. Space (as three anisotropic dimensions) and time (as three well-ordered dimensions nominated as "past, present, future") can give an example of that complementary independence in the finite case. Consequently the true notion of space-time involves already, though implicitly, the above finite invariance to the axiom of choice.

A more general viewpoint is possible as to the relation between one and the same, but described in two different kinds of terms: those of quantum mechanics, on the one hand, and those of general relativity, on the other hand:

The "atom" of that sameness is the qubit, a three-dimensional unit ball as its three dimensions can be in a few different interpretations:

$\left(\mathrm{A}^{\prime}\right)$ as two values of the same quantity and the value of the energy $\mathrm{E}_{\mathrm{A}}$ ' causing the change from the one to the other.

(B') as two values of two conjugate quantities and value of the energy $\mathrm{E}_{\mathrm{B}}$ ' causing the 
change from the one to the other.

(C') as two probabilities of the same quantity and the value of the energy $\mathrm{E}_{\mathrm{C}}$ ' causing the change from the one to the other.

(D') as two probabilities of two conjugate quantities and the value of the energy $\mathrm{E}_{\mathrm{D}}$ ' causing the change from the one to the other.

The quadrangle A'B'C'D' is a new interpretation (in terms of $Q M$ ) of the above quadrangle ABCD.

$E_{A}$ ' is "inertial energy", i.e. the energy, which corresponds to "inertial mass", and then $\mathrm{E}_{\mathrm{A}}$ ' should be "gravitational energy" corresponding to gravitational mass.

However $E_{C}$, and $E_{D}$ ' would be a new kind of energy, which corresponds to information. Two kinds of such information might be distinguished and nominated inertial and gravitational information in analogy to the corresponding kinds of mass.

Indeed both $\mathrm{E}_{\mathrm{C}}$, and $\mathrm{E}_{\mathrm{D}}$ ' cause some change in probability and consequently make sense as information. As both are the values of probability, it is a dimentionless number. An exact energy corresponds to it one-to-one: It is proportional to it by means of the Planck constant per second. Informational energy would be the natural name for it and could be divided correspondingly into gravitational and inertial one.

Some corresponding informational energy can be ascribed to any informational process including e.g. those in our PCs. The corresponding energies are incredibly small for the value of the Planck constant per second is as incredibly small. However the informational processes, which mankind at present can create and manage are different in principle from those in the nature at least for their informational energy, which is comparable with their mass energy.

The difference consists in the absence of any well-ordering as to quantum-informational processes. Mass energy, which we know well as gravitational as inertial, can be obtained from informational energy after any well-ordering e.g. that caused by any representation in space-time.

Only the triangle ABC (A'B'C') has been considered by now, after which some doubling of the point B (B') has to be ascertained. The natural is the "second" point B to be identified as the point $D$ of the quadrangle $A B C D$ (A'B'C'D'). Doing so, entanglement and gravity are identified, too, as the mathematical and the physical image of one the same, and the gravitational constant is what allows of them to coexist peacefully.

One can consider the different particular cases of the quadrangle ABCD (A'B'C'D') one by one after some of them have already been mentioned:

The first group of particular cases comprises the coincidences of some of the points A, B, C, and D (A', B', C', and D'):

The most particular case is the coincidence of all the points $\mathrm{A}, \mathrm{B}, \mathrm{C}$, and D. It corresponds to the three principles of general relativity as they were formulated by Einstein in 1918 and to the revised equation involving the suitable nonzero cosmological constant.

The next particular case is the degeneration of $A B C D$ ( $A^{\prime} B^{\prime} C^{\prime} D^{\prime}$ ') to the segment $A B$ $\equiv \mathrm{CD}\left(\mathrm{A}^{\prime} \mathrm{B}^{\prime} \equiv \mathrm{C}^{\prime} \mathrm{D}^{\prime}\right)$. It corresponds to the initial form of the Einstein equation without the 
cosmological constant and without the "Mach'sches Prinzip".

The quadrangle $\mathrm{ABCD}$ ( $\mathrm{A}^{\prime} \mathrm{B}$ 'C'D') can degenerate to a triangle if any two of the four points coincide. This might take place if only one of the three Einstein principles (1918) holds, which would call the coincidence of $\mathrm{A}$ and $\mathrm{B}, \mathrm{C}$ and $\mathrm{D}$ as well as $\mathrm{A}$ and $\mathrm{C}$ accordingly. If (B and C) or (A and D) coincide, this seems to lead to the case of the segment in physical considerations. As to the eventual coincidence of $\mathrm{B}$ and $\mathrm{D}$, this would exclude the option of entanglement in principle and could be reckoned as an implicit Einstein principle, too, formulated as the conjecture of "hidden variables" (or "hidden parameters") in quantum mechanics (e.g. Einstein 1957, pp. 82-86), though.

The second group of interesting particular cases comprises those of equation for some of the sides of quadrilateral $\mathrm{ABCD}$ (A'B'C'D').

The quadrilateral $\mathrm{ABCD}$ is "contravariant" while $\mathrm{A}^{\prime} \mathrm{B}{ }^{\prime} \mathrm{C}^{\prime} \mathrm{D}$ ' is "covariant": $\mathrm{ABCD}$ refers to the results of measuring while $\mathrm{A}^{\prime} \mathrm{B}^{\prime} \mathrm{C}^{\prime} \mathrm{D}$ ' refers to the measures:

Mechanical movement is usual to be thought "contravariantly", i.e. as the movement of a part to the rest of the whole. However it can be always interpreted "covariantly", which means a general and uniform movement of all parts to all rest parts according to a corresponding change in measure visible as that general movement. The obvious example of the latter is the expansion of the universe.

How do the former and the latter kind of mechanical movement refer to each other? Is there a common viewpoint, from which they can be thought as equivalent?

At first glance their eventual equivalence seems nonsense even merely for the huge difference in energy. However this merely means that the movement per a unit of mass, i.e. its kinematic representation should consider rather than the usual dynamic one. Reversely, taking for granted that equivalence, then the quantity of mass as well as the force of gravity can be reckoned as a suitable addition for the corresponding transition to the standard dynamical approach:

One can see this transition in terms of part and whole: The dynamical approach is according to the part while the kinematic one to the whole and the force of gravity is necessary to equate the two viewpoints to each other.

Another measure of the relation of part and whole is probability, which can be thought as standard as quantum. Being standard, the phenomena of entanglement cannot arise: They are specific for quantum probability (i.e. normed QM). As above, gravity is what should discuss as another viewpoint to entanglement, namely which is well-ordered in space-time: It arises for the transition to QM (or to quantum probability):

This implies: Mass is entanglement per a unit of time and both of them are energies: accordingly that of the smooth (continuous) movement seen dynamically and that of the discrete movement (quantum leap) seen kinetically. Gravity is what should add to equate both the viewpoints to each other.

One can interpret the separated equations of sides representing different particular cases of the quadrilateral $\mathrm{ABCD}$ (or seen "covariantly", $\mathrm{A}^{\prime} \mathrm{B}^{\prime} \mathrm{C}^{\prime} \mathrm{D}^{\prime}$ ):

The vertical sides $\mathrm{AC}\left(\mathrm{A}^{\prime} \mathrm{C}^{\prime}\right)$ and $\mathrm{BD}$ (B'D') correspond to the inertial mass and to 
the forces changing the state of mechanical movement as they cause some acceleration or deceleration. The side $\mathrm{AC}\left(\mathrm{A}^{\prime} \mathrm{C}^{\prime}\right)$ can be linked to the space-time position of the body with that inertial mass, and the side BD (B'D) accordingly, with the energy of the body. Consequently their eventual equation as a particular case would mean the state of inertial movement, or the absence of any force acting over the body. The conception of 'body' supposes for it to be able to be rigorously distinguished from its environment: One can say unambiguously whether a space-time point belongs to the body or to the environment. The choice between the body and the environment must be disjunctive. Consequently any energy can belong either to the body according its inertial mass or to the force acting from the environment to the body. The equation of $\mathrm{AB}\left(\mathrm{A}^{\prime} \mathrm{B}^{\prime}\right)$ and $\mathrm{CD}$ ( $\left.\mathrm{C}^{\prime} \mathrm{D}^{\prime}\right)$ means the equilibrium between the body and environment.

One can thought of the horizontal sides $\mathrm{AB}$ (A'B') and $\mathrm{CD}$ (C'D') to some extent in analogy with the vertical sides. The difference between the two cases consists in the difference between 'body' and 'field'. The "field" in terms of 'body' represents the intersection between a body and its environment. Consequently the horizontal sides represent the inseparability of the body from its environment while the vertical ones, accordingly their separability. The 'field' is necessary to describe that inseparability as a function of space-time coordinates. So, 'gravitational field' is an expression established in tradition for the mechanical "field" in the sense above. It originates from the entanglement of the body with its environment. The eventual equation of the horizontal sides $\mathrm{AB}\left(\mathrm{A}^{\prime} \mathrm{B}^{\prime}\right)$ and $\mathrm{CD}$ ( $\mathrm{C}^{\prime} \mathrm{D}^{\prime}$ ) would mean that the acting gravitational field is constant since the degree of entanglement is constant. If entanglement energy is introduced in a suitable way, then the equation above could be represented more exactly as the equation of gravitational and entanglement energy. The relevant approach for introducing the entanglement energy is by means of the mutual entropy of the body and its environment per a unit of time.

The first two Einstein principles (1918) have been above interpreted as the coincidence of $\mathrm{AC} \equiv \mathrm{BD}$. This is consistent with the present consideration by means of the quadrangle $\mathrm{ABCD}$ (and $\mathrm{AB}=\mathrm{CD} \neq 0$ ) for the equation of gravitational and inertial mass, which equates unavoidably gravitational and inertial energies and forces, too. This means that the dimension, in which the sides $\mathrm{AB}$ and $\mathrm{CD}$ can be situated, is merely absent in general relativity. In fact, it can arise only after quantum mechanics has introduced the complementarity of the sides $\mathrm{AC}$ and BD. However general relativity represents equivalently any "length" in that dimension by the curvature of space-time.

The third group of particular cases is that of one or more right angles in the quadrangle $\mathrm{ABCD}$ ( $\mathrm{A}^{\prime} \mathrm{B}^{\prime} \mathrm{C}^{\prime} \mathrm{D}^{\prime}$ ). The sense is some two sides (e.g. those are the sides $\mathrm{AB}$ and $\mathrm{AC}$ for the angle A) to be independent of each other. An eventual dependence would mean that there exist an entity such that it is both a body and a field or both a body and its environment. Wave-particle duality states the former, and entanglement the latter.

Since the notion of kinetic energy is linked to the movement of a body, and that of potential energy to a field (or the body's environment), the energy of a quantum entity ("particle") is both potential and kinetic, the energy of entanglement as well. 
The Bekenstein bound can be considered in a generalized way, as a link between QM and LM: For that a notion of measure temperature should introduce as the ratio of LM to QM. In terms of LM, it would represent the curvature of LM. Given a LM and its curvature, QM can be uniquely restored. The dimensions of QM are always three, but those considerations need refinement for the arbitrary number of dimensions as to LM:

Fortunately, the physically meaningful case is three-dimensional: LM and the ratio of LM to QM can be obtained immediately. As to the general case, there are possible different approaches. Two groups of them seem obvious: The one reduces an arbitrarily dimensional LM to three-dimensional LM, and the other introduces QM for each dimension of LM. This is out of the present consideration.

One can pay attention that the Boltzmann rather than gravitational constant appears in the mathematical formulae for the Bekenstein bound. This imposes to be elucidated the relation between those two constants in the present context:

Both connect the absence of well-ordering in principle with a given well-ordering in space-time. Consequently they are linked to the axiom of choice and can be naturally interpreted as the constants of physical choice.

They are distinguished from each other as follows:

The gravitational constant is "kinematic" and "covariant": It defines a velocity per a unit of mass (energy) and distance and the properties of the space-time as a whole or of any whole in general.

Accordingly the Boltzmann constant complements it as its "dynamic" and "contravariant" counterpart. It defines a value of energy per a unit of temperature or per a unit of energy (mass) in a value of entropy. Consequently, the Boltzmann constant is which determines the properties of an entities within the space-time and locally.

The gravitational constant and the Boltzmann constant can refer accordingly to each of the two components of quantum temperature, which can be thought as the kinematic and contravariant part and as the dynamic and covariant part correspondingly.

The conclusion is that general relativity is a thermodynamic theory of a certain and rather extraordinary kind. Its features are:

It describes the thermodynamic properties of the whole seen insides, from the viewpoint of its parts as a force field, namely the gravitational field.

Those thermodynamic properties are quite different from the ones of a classical statistical ensemble for quantum probability as normed QM rather than classical probability and the corresponding normed $\mathrm{BM}$, from which it originates.

Unlike the classical probability, the quantum one is very closely connected to the properties of space-time and the behavior of all the entities within it. That connection is what is discussed in fact and generalized as the theory of gravity in general relativity.

The notion of quantum temperature is what is called upon to bare that hidden thermodynamic essence both of gravity and general relativity as a correct theory of it. It is what allows of the Boltzmann and gravitational constant to be considered together and in tight 
connection as above.

The gravitational constant requires an effectively acting force field necessarily to exist for any nonzero energy by means of some acceleration corresponding one-to-one to that energy in a certain value of velocity.

The Boltzmann constant accordingly generates some entities defined as such by the corresponding energy of that temperature, on which the above force field can already act.

The "Big Bang" turns out to be a kind of projection of the real state of affairs in terms of LM and BM. More exactly, it reproduces the picture as if QM could reduce comprehensively to two groups of LM (or BM) or as if the genesis of the universe could do to two unrelated groups of phenomena: on the one hand, thermodynamic, and then gravitational (mechanical) ones, on the other hand.

The "Big Bang" represents a picture of an initial colossal explosion, the consequences of which we can observe and explain in terms and laws of the well-known physics. The problem how could happen so extraordinary event as the "Big Bang" is merely circumvented and postponed to the future. The convenience of the "Big Bang" lies in the fact that it can hold and thus hide any embarrassing questions at a time that is beyond the horizon of human knowledge.

The mechanical and gravitational phenomena are placed in the foreground, and the thermodynamic ones remain in the background in the illustration known as the "Big Bang". One might move its foundations as an initial step simply swapping the background and foreground, that is, as the picture looks backwards:

This would mean to represent the genesis of the universe as the observed cooling in temperature (yet the ordinary rather than quantum one) in a constant energy and in a found, then already available somehow space-time, or in other words, the explosion to be only in entropy. Since the different space-time positions mean also different states, the increase of entropy would require a corresponding space-time expansion restricted by gravity. The nature of that informational "demon" that can summon all the variety of the universe in a limited number of states and thus to increase its temperature adiabatically is not less embarrassing than the true "Big Bang”.

QM suggests and even requires in a sense the unity (also equivalence, invariance, relativity) of three aspects, which otherwise are absolutely independent in any theory of measurement founded on LM and BM: Those are the discrete, continuous (smooth), and probabilistic. Being independent classically, each of them needs its proper cause to take place, and can or cannot be a cause of any of the rest. For example, the "Big Bang" orders them in following way. It happened (being a form of the discrete) first, causing a continuous (smooth) constant and even perhaps eternal expansion in space-time. Any subset of that expanding space-time represents a statistic ensemble and generates probabilities, which can be only statistical physically. So the discrete ("Big Bang") has not any cause and allows of taking place for a creator or demon to generate it.

QM requires only a change in human thinking: Is there any of the three aforesaid aspects, then the rest two are available necessarily just for QM. Since the expansion of the 
universe is an unquestionable fact, the discrete and probabilistic must be available obligatorily without any other cause. They are not caused by the expansion in any classical meaning of "cause", but are necessary available being its complementary aspects, though. As to time, it refers only to the continuous aspect, which, however, already is not universal physically within the QM. For that, the question when (i.e. to be chosen a moment in time) the discrete or the probabilistic has taken, takes, or will take place is meaningless: No need of any creators or demons they to happen, but they do not happen in time. If one wishes some illustration of the way of their happening in relation to time, they can be thought as points out of time, but not as ones before or after the time, and even less within it. If one wants at any cost to situate them anywhere but within the time, another kind of that illustration might be possible anyway: Both the discrete and the probabilistic take place in any instant of time.

One can object that the question when a quantum leap is taking place is absolutely meaningful. This is so, but only if that quantum entity, which is jumping, is a part of a system, which to comprise it, e.g. the apparatus in quantum mechanics. As to the universe, one needs any item, which to include the universe as a part of its. This would generate a "bad infinity" of uniformly following questions: In other words the answer is postponed for and instead of being given. Instead of that one can postulate the universe as what is not a part of anything besides of itself (it can be a real part of itself). In fact the universality of QM is consistent only with that postulate.

Another objection is that one can project the observed expansion back in time to a beginning when that expansion has started. However that projection is only possible under the condition that the time velocity has been constant all the time, which is equivalent to energy conservation: This means that an energy corresponding to a frequency in a given past moment of time is the same with those in any other moment. This is not a fact, which can be observed directly: Rather the observed expansion of the universe contradicts it implicitly. Anyway they can be reconciled at the cost of the Big Bang. Getting rid of them is much more natural, though. In fact, QM in general is inconsistent with energy conservation since the latter reduces it to LM (BM).

Consequently, QM requires energy conservation to be sacrificed:

Time and energy being two conjugate quantities should be independent of each other: However energy conservation requires a one-to-one mapping between them (Noether 1918). Given energy conservation, QM is necessarily reduced to a one-dimensional LM (BM), or in other words, to the degenerate case of a segment on the energy-time plane.

QM requires to get rid of energy conservation also for removing the Big Bang. The entire energy of the universe must appear gradually as to its continuous (smooth) image in space-time. This can happen if and only if a new additional portion of energy sources from nothing at each moment of time. One can suggest a kind of positive feedback, which constantly involves energy.

Dark energy and matter can be considered as an indirect evidence of the fact that some quantity of energy appears continuously. Indeed it should be "dark" in principle 
originating from the nothing.

The mechanism of such a positive feedback, which to generate energy always, is not too difficult to be conjectured, even more than one:

A first example can be the as static as the dynamic (even better) Casimir effect as to the second as to the first (even better) quantization. Some of those forecast effects are observed experimentally (Lamoreaux 1997; Wilson et al. 2011). The essence of all of them is the distinction in the form of space-time estrangement (moving away) of two conjugate quantities or of quantum pairs, e.g. such as virtual pairs generated from the vacuum. That estrangement proves the physical existence of both dual Hilbert spaces after they can be moved off from each other in an experimentally observable way. For example a movement with a speed close to the light velocity can produce such an estrangement as to the dynamical Casimir effect for the second quantization (Wilson et al. 2011). Right a fast enough expansion could generate that space-time estrangement and thus energy, namely the energy of the two moving away virtual particles, which do not yet compensate mutually.

Besides, QM can conjecture a more immediate mechanism to generate energy from nothing. One of its hypostases, that which cannot be well-ordered in principle and is probabilistic, merely a number without any physical dimension, turns out to possess energy for the invariance to well-ordering after doing it in time. The positive feedback could be the following: The expansion of space-time means two things: The one is that the entropy of the space-time points increases, and the other is that the energy-impulse of the universe has to increase, too, as the reciprocal (conjugate) of space-time. If the entropy increases and this is a natural law, it requires for the corresponding energy to increase. However since one observes a constant drop in temperature, this means that the entropy has increased more, generating accordingly a new increase in energy-impulse, and all is repeated again and again.

In other words, the expanding universe, increasing entropy, and dropping temperature are consistent with each other, but inconsistent with energy conservation just as QM is inconsitent with it. The nothing, numbers, or probabilities transform themselves into energy, i.e. into something continuously with a rate determined by the fundamental constants. This needs no creator or demons since it is a natural law. QM unlike LM (BM) is just what allows of it to hold. The notion of quantum temperature aids this to be seen. Dark energy and matter might be those which have not been yet transformed into "visible" things, but otherwise their values are "already" fixed by the corresponding discrete leap aka the "Big Bang". "Already" is in quotation marks for the "Big Bang" should see out of time as this is explained above.

Of course, the objectivity of the text is philosophical. The invention of cleverer and realer positive feedbacks for the universe to arise in a natural law should remain to the mathematicians and physicists.

QM suggests for the mathematical and physical not to be separated any more. This has at least a few important philosophical consequences or generalizations:

There exists the opportunity for a mathematical model to be an absolutely exact copy of physical reality. 
The ideal and real can pass each other immediately or in other words, transform into each other.

The psychophysical problem has a simple and natural solving: The psychical possesses have physical dimensions such as energy and thus can manage physical processes immediately. That problem is only an epiphenomenon of the classical measure theory.

Any entity has a very complicated hidden structure as the measure of which is its energy corresponding to a certain quantity of quantum information per a unit of time. If one starts to change that internal build in a suitable way, the entity can be transformed directly into another, even into any other one. Any physical entity represents its own description, design, something like drawings to be created or modified. All physics ever and so far has considered any entity as a kind of black box studying only its "transfer function" which connects all the set of possible input physical quantities with that of output ones as a function according a natural law. Now one got the chance to look at it and at its concealed structure and method of operation, which turn out to be informational, but quantum-informational rather than classical-informational.

\section{The perspective:}

Is there any measure more general and universal than QM? If there is, it could be called "generalized quantum measure" (GKM). According to current knowledge, we cannot even figure what might cause such a measure to be introduced or what would constitute.

One can postulate the absolute universality of QM. This implies a series of philosophical conclusions and new interpretations of well-known facts. The most of them have been already mentioned in a slightly different context above. What is worth to emphasize here is the following:

A universal measure as QM suggests that all entities are not more than different forms of a substance shared by all of them as their fundament: It is quantum information and represents a general quantity, which is both mathematical and physical in its essence. The longstanding philosophical idea of a single and general substance can be already discussed in terms of exact science. 


\section{RE F E R E N C ES :}

ALEXANDROFF, P. (1916) Sur la puissance des ensembles measurable (B). Comptes rendus hebdomadaires des séances de l'Académie des sciences, 162 (28.02.1916), pp. 323-325.

ASPECT, A., GRANGIER, R., and ROGER, G. (1981) Experimental tests of realistic local theories via Bell's theorem. Physical Review Letters, 47 (7), pp. 460-463.

ASPECT, A., GRANGIER, R., and ROGER, G. (1982) Experimental Realization of Einstein-Podolsky-Rosen-Bohm Gedanken Experiment: A New Violation of Bell's Inequalities. Physical Review Letters, 49 (2), pp. 91-94.

AVRON, J. and ELGART, A. (1999) Adiabatic Theorem without a Gap Condition. Communications in Mathematical Physics, 203 (2), pp. 445-463.

BANACH, S. and TARSKI, A. (1924) Sur la decomposition des ensembles de points en parties respectivement congruentes. Fundamenta Mathematicae, 6 (1), pp. 244-277.

BEKENSTEIN, J. (1972) Black Holes and the Second Law. Nuovo Cimento, 4 (15/ 12.08.1972), pp. 737-740.

BEKENSTEIN, J. (2003) Information in the Holographic Universe. Scientific American, 289 (2/ August), pp. 58-65.

BEKENSTEIN, J. (2005) How does the entropy/ information bound work? Foundations of Physics, 35 (11/November), pp. 1805-1823.

BOHR, N., KRAMERS, H. and SLATER, J. (1924) The quantum Theory of Radiation. Philosophical Magazine Series 6, 47 (281), pp. 785-802.

BELL, J. (1964) On the Einstein - Podolsky - Rosen paradox. Physics (New York), 1 (3), pp. 195-200.

BORN, M. (1926a) Zur Quantenmechanik der Stoßvorgänge. Zeitschrift für Physik, 37,(12/ December), pp. 863-867.

BORN, M. (1926b) ; Quantenmechanik der Stoßvorgänge. Zeitschrift für Physik, 38 (11-12/ November), pp. 803-827.

BORN, M. (1927a) Das Adiabatenprinzip in der Quantenmechanik. Zeitschrift für Physik, 40, (3-4/ März), pp. 167-192.

BORN, M. (1927b) Physical aspects of quantum mechanics. Nature, 119 (05.03.1927), pp. 354-357.

BORN, M. (1954) 'The statistical interpretation of quantum mechanics' [Online] Nobel Lecture, December 11, 1954. Available from: http://nobelprize.org/nobel_prizes/physics/laureates/1954/ born-lecture.pdf .

BORN, M. and FOCK, V.. (1928) Beweis der Adiabatensatzes. Zeitschrift für Physik A: Hadrons and Nuclei, 51 (3-4), pp. 165-180.

CARATHÉODORI, C. (1956) Mass und Integral und ihre Algebraisierung. Basel: Birkhäuser.

CLAUSER, J. and HORNE, M. (1974) Experimental consequences of objective local theories. Physical Review D, 10 (2), pp. 526-535.

DIRAC, P. (1928) The Quantum Theory of the Electron. Proceedings of the Royal Society A: Mathematical, Physical \& Engineering Sciences, 117 (2 /01.02.1928), pp. 610-624. 
DIRAC, P. (1930) A Theory of Electrons and Protons. Proceedings of the Royal Society A: Mathematical, Physical \& Engineering Sciences, 126 (2/ 01.02.1930), pp. 360-365.

DIRAC, P. (1931) Quantized Singularities in the Electromagnetic Field. Proceedings of the Royal Society A: Mathematical, Physical \& Engineering Sciences, 133 (9/01.09.1930), pp. 60-72.

EINSTEIN, A. (1910) Theorie der Opaleszenz von homogenen Flüssigkeiten und Flüssigkeitsgemischen in der Nähe des kritischen Zustandes. Annalen der Physik, Vierte Folge, 33 (338/16), pp. 1275-1298.

EINSTEIN, A. (1917) Kosmologische Betrachtungen zur allgemeinen Relativitättheorie. In: Sitzungsberichte der Königlich preussischen Akademie der Wissenschaften, Erster Halbband, VI. Berlin: Verlag der Königlichen Akademie der Wissenschaften, pp. 142-152.

EINSTEIN, A. (1918) Prinziplelles zur allgemeinen Relativitätstheorie. Annnalen der Physik, 55 (4), pp. 241-244.

EINSTEIN, A., PODOLSKY, B., and ROSEN, N. (1935) Can Quantum-Mechanical Description of Physical Reality Be Considered Complete? Physical Review, 47, (10/15.10.1935), pp. 777-780.

EINSTEIN, A. (1957) Autobiographishes. In: SCHLIPP, P. (ed.) Albert Einstein: PhilosopherScientist. New York: Tudor Publishing Company, pp. 1-95.

FIERTZ, M. (1939) Über die relativistische Theorie kräftefreier Teilchen mit beliebigem Spin. Helvetica Physica Acta, 12 (1), pp. 3-17.

GAMOV, G. (1970) My World line: An Informal Autobiography. New York: The Viking Press.

HAUSDORF, F. (1916) Die Mächtigkeit der Borelschen Mengen. Mathematische Annalen, 77 (3), pp. 430-437.

HEISENBERG, W. (1925) Über quantentheoretische Umdeutung kinematischer und mechanischer Beziehungen. Zeitschrift für Physik, 33 (1), pp. 879-893.

JACOBSON, T. (1995) Thermodynamics of Spacetime: The Einstein Equation of State. Physical Review Letters, 75 (7/ 14.08.1995), pp. 1260-1263.

KOCHEN, S. and SPECKER, E. (1968) The problem of hidden variables in quantum mechanics. Journal of Mathematics and Mechanics, 17 (1), pp. 59-87.

LAMOREAX, S. (1997) Demonstration of the Casimir Force in the 0.6 to $6 \mu \mathrm{m}$ Range. Physical Review Letters, 78 (1/ 06.01.1997), pp. 5-8.

LÖWENHEIM, L. (1915) Über Möglichkeiten im Relativkalkül. Mathematische Annalen, 76 (4), pp. 447-470.

NEUMANN, J. (1932) Mathematische Grundlagen der Quantenmechanik. Berlin: Verlag von Julius Springer.

NOETHER, E. (1918) Invariante Variationsprobleme. In: Nachrichten von der Gesellschaft Wissenschaften zu Göttingen, Mathematisch-physikalische Klasse (1918). Berlin: Weidmannsche Buchhandlung, pp. 235-257.

PAULI, W. (1940) The Connection Between Spin and Statistics. Physical Review, 58 (8/ October), pp. 716-722. 
SCHRÖDINGER, E. (1926a) An Undulatory Theory of the Mechanics of Atoms and Molecules. Physical Review, 28 (6), pp. 1049-1070.

SCHRÖDINGER, E. (1926b) Über das Verhältniss der Heisenberg-Bor-Jordanischen Quantenmechanik zu der meinem. Annalen der Physik, 384, (8), pp. 734-756.

SKOLEM, T. (1919a) Untersuchung über die Axiome des Klassenkalküls und über Produktations- und Summationsprobleme, welche gewisse Klassen von Aussagen betreffen. Skrifter utgit av Videnskabsakademiet i Kristiania, I (3), pp. 37-74.

SKOLEM, T. (1919b) Logisch-kombinatorische Untersuchungen über die Erfüllbarkeit und Beweisbarkeit mathematischen Sätze nebst einem Theoreme über dichte Mengen. Skrifter utgit av Videnskabsakademiet i Kristiania, I, (3), pp. 1-36.

SKOLEM, T. (1923) Einige Bemerkungen zur axiomatischen Begründung der Mengenlehre. In: Matematikerkongressen $i$ Helsingrofs den 4-7 Juli 1922, Den femte skandinaviska matematikerkongressen, Redogörelse. Helsinki: Akademiska-Bokhandeln, pp. 217-232.

SIERPIŃSKI, W. (1924) Sur la puissance des ensembles measurable (B). Fundamenta Mathematicae, 5 (1), pp. 166-171.

SMOLIN, L. (2002) Three Roads to Quantum Gravity. New York: Basic Books.

Weber, H. (1893) Leopold Kronecker. In: Jahresbericht der Deutschen Mathematiker-Vereinigung, 2 (1891 - 1892), pp. 5-31.

VITALI, G. (1905) Sul problema della misura dei gruppi di punti di una retta. Bologna: Gamberini and Parmeggiani.

WILSON, C. et al. (2011) Observation of the dynamical Casimir effect in a supeconducting circuit. Nature, 479 (7373/ 17.11. 2011), pp. 376-379. 